Rev. Biol. Neotrop. 10(1): 28-35. 2013

\title{
ERMINAÇÃO DE SEMENTES DE TRÊS ESPÉCIES DO GÊNERO PARKIA SUBMETIDAS A DIFERENTES MÉTODOS DE SUPERAÇÃO DE DORMÊNCIA E TEMPERATURA
}

\author{
Fabieli Pelissari \\ Universidade Federal de Mato Grosso, Campus de Sinop, Mestranda do Programa de Ciên- \\ cias Ambientais, Instituto de Ciências Naturais, Humanas e Sociais, Sinop, Mato Grosso, \\ Email: fabieli_ufmt@yahoo.com.br
}

\section{Célio Jacinto da Silva}

Universidade Federal de Mato Grosso, Campus de Sinop, Instituto de Ciências Agrárias e Ambientais, Sinop, Mato Grosso. Email: celiojs_ufmt@yahoo.com.br

\section{Carlos Vinicio Vieira}

Universidade Federal de Mato Grosso, Campus de Sinop, Instituto de Ciências Agrárias e Ambientais, Sinop, Mato Grosso. Email: vieiracv@gmail.com

\begin{abstract}
Resumo: O trabalho teve como objetivo avaliar métodos de superação de dormência em diferentes temperaturas em sementes de Parkia pendula, Parkia platycephala e Parkia multijuga, visando a maximização e uniformização da germinação. As sementes de cada espécie passaram pelos seguintes tratamentos: T1 - escarificação mecânica em esmeril elétrico; T2 - escarificação mecânica em esmeril elétrico seguido por imersão em água corrente por 24h; T3 - escarificação com ácido sulfúrico 98\% por $10 \mathrm{~min}$.; T4 - imersão em água a $80^{\circ} \mathrm{C}$ por $10 \mathrm{~min}$.; T5- controle. As sementes de cada tratamento foram acondicionadas em germinadores do tipo B.O.D. em temperaturas de $25^{\circ} \mathrm{C}, 30^{\circ} \mathrm{C}$ e $25-30^{\circ} \mathrm{C}$ com fotoperíodo de 12 horas. Todas as espécies apresentaram interação entre os dois fatores estudados (superação de dormência e temperatura), ou seja, existe pelo menos uma combinação ideal entre esses fatores que otimiza a germinação e o aumento no IVG, com exceção da porcentagem de germinação para $P$. multijuga. Para superação da dormência de $P$. pendula e $P$. platycephala recomenda-se o T1 e para $P$. multijuga o $\mathrm{T} 2$. A temperatura de $30^{\circ} \mathrm{C}$ é recomendada para as três espécies.
\end{abstract}

Palavras-chave: dormência, escarificação, germinação, temperatura.

\begin{abstract}
The study aimed to overcome dormancy methods at different temperatures of Parkia pendula, Parkia platycephala and Parkia multijulga seeds, to increase and standardize germination. The seeds of each species were submitted following treatments: T1 - scarification on electric emery; T2 scarification on electric emery followed by immersion in water for 24 hours, T3 - with sulfuric acid $98 \%$ for $10 \mathrm{~min}$; T4 - immersion in water $80^{\circ} \mathrm{C}$ for $10 \mathrm{~min}$; T5- control. The seeds of each treatment were placed in germination of $\mathrm{BOD}$ at temperatures of $25^{\circ} \mathrm{C}, 30^{\circ} \mathrm{C}$ and $25-30^{\circ} \mathrm{C}$ with a photoperiod of 12 hours. All species showed statistical interaction between the two factors studied (overcoming dormancy $X$ temperature), except for the germination of $P$. multijuga, therefore, there is at least an ideal combination of these factors that optimizes germination and increase in IVG. For overcome dormancy $P$. pendula and $P$. platycephala recommend the T1, and to $P$. multijuga the T2. The temperature of $30^{\circ} \mathrm{C}$ is recommended for all three species.
\end{abstract}

KEY WORDS: dormancy, scarification, germination, temperature. 


\section{INTRODUÇÃO}

$\mathbf{N}$ as últimas décadas é considerável o aumento na recuperação de áreas degradadas e restabelecimento de florestas. Instituições como o Ministério do Meio Ambiente (MMA), a Organização Mundial para Agricultura e Alimentos( "FAO"), o Banco Internacional para a Reconstrução e Desenvolvimento (Bird), juntamente com o Programa de Recuperação de Áreas Degradadas na Amazônia (Pradam), buscam através de várias ações contribuir para a recuperação de áreas degradas. O Brasil possui a sexta maior área reflorestada do mundo (Bacha, 2008) e de acordo com o Ministério da Agricultura e Pesquisa Agropecuária (2013) recuperar 15 milhões de hectares de áreas degradadas entre os anos de 2010 e 2020 é uma das metas para o programa do governo federal de redução da emissão de gases de efeito estufa.

Esses dados estimulam cada vez mais as pesquisas na área de produção de mudas nativas, e dentre estas se encontra a tecnologia e desenvolvimento de sementes, uma vez que há uma grande lacuna no conhecimento sobre o comportamento biológico de muitas dessas espécies, como de padrões para estabelecimento de sua comercialização (Melo et al, 2011). A semente é a principal forma de propagação das espécies, em especial, das florestais, sendo considerada também mais fácil e econômica comparando-se com a propagação vegetativa e com a micropropagação (Pereira et al, 1995). Sementes de qualidade que apresentam características como alta porcentagem de germinação, sincronia, rapidez no desenvolvimento e alta sobrevivência são indispensáveis para que a produção seja economicamente viável (Pinedo \& Ferraz, 2008).

O processo de germinação da semente tem início com a absorção de água através da embebição (Finch-Savage \& Leubener-Metzger, 2006). Esta absorve a água e seus tecidos tornam-se túrgidos, o tegumento hidratado amolece e se rompe (Floriano, 2004). Esse processo é influenciado pela disponibilidade de água, temperatura, substrato, luz e a ausência de patógenos (Schmi$\mathrm{dt}, 2000$ ). Porém, algumas sementes, mesmo em condições favoráveis não germinam, sendo então denominadas dormentes, ou seja, apresentam alguma restrição interna ou sistêmica à germinação, que deverá ser superada para que o processo germinativo ocorra (Cardoso, 2004). A dormência impede a germinação, porém é uma adaptação para a sobrevivência da espécie a longo prazo, fazendo com que as sementes se mantenham viáveis por maior período de tempo (Floriano, 2004).

A semente pode estar dormente devido os tecidos que a envolvem, exercendo certa impermeabilidade a água, conhecida como dormência tegumentar, sendo a mais comum das categorias (Fowler \& Bianchetti, 2000).

O gênero Parkia apresenta uma grande diversidade na Amazônia, com árvores de grande por- te que ocorrem na floresta de terra-firme, várzea sazonal, floresta secundária, e no norte da América do Sul, onde existem 17 espécies (Hopkins, 1986), sendo utilizadas frequentemente em reflorestamento (Miranda et al, 2012; Melo et al 2011; Rosseto et al, 2009; Galeão et al, 2006; Oliveira et al, 2006).

As sementes deste gênero apresentam dormência física, como relatado por Varela et al (1987), Fowler \& Bianchetti (2000), Oliveira et al (2006) e Pereira \& Ferreira (2010), sendo utilizados métodos químicos e físicos para a superação da dormência. Tratamentos com ácido sulfúrico apresentaram sucesso nas espécies Enterolobium contortisiliquum (Eira et al, 1993), Piptadenia moniliformis (Azeredo et al, 2010), Bauhinia monandra (Alves et al, 2000), Dimorphandra mollis (Hermansen et al, 2000) e Parkia nitida (Cruz et al, 2001).

Sementes das espécies de Mimosa foliolosa (Silveira \& Fernandes, 2006), Caesalpinia pyramidalis (Alves et al, 2007) e Acacia caven (Escobar et al, 2010) tiveram a dormência superada através da escarificação mecânica. No entanto, a água quente foi mais favorável para as espécies Mimosa bimucronata (Ribas et al, 1996) e Ochroma pyramidales (Netto, 1994).

Outro fator determinante na germinação das sementes é a temperatura, onde faixas de temperatura ideal determinarão o máximo de germinação no menor espaço de tempo possível (Carvalho \& Nakagawa, 2000). De acordo com Albrecht et al (1986) as temperaturas entre $26,5^{\circ} \mathrm{C}$ e $35^{\circ} \mathrm{C}$ são mais favoráveis para a germinação das sementes. Porém, esses valores podem variar de acordo com a região de ocorrência de cada espécie.

A aplicação e a eficiência dos tratamentos para superação da dormência dependem do grau de dormência, que é variável entre diferentes espécies (Oliveira et al, 2003), sofrendo influência da temperatura de acordo com a região de ocorrência. Por isso a importância em avaliar diferentes métodos de superação de dormência de sementes de espécies de Parkia nas diferentes temperaturas. Diante do exposto, este trabalho teve como objetivo avaliar métodos de superação de dormência em diferentes temperaturas de sementes de Parkia pendula (Willd.) Benth. ex Walp., Parkia platycephala Benth. e Parkia multijuga Benth., visando aumentar e uniformizar a germinação.

\section{Material e Métodos}

A identificação botânica do material foi realizada pelo Herbário Centro Norte Mato Grossense - CNMT. Os frutos de Parkia pendula, Parkia multijuga e Parkia platycephala foram coletados no município de Sinop - MT, no período de outubro a dezembro de 2011,com coordenadas geográficas

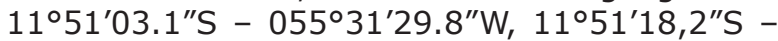
$055^{\circ} 30^{\prime} 38.2^{\prime \prime} \mathrm{W}, 11^{\circ} 52^{\prime} 01.8^{\prime \prime} \mathrm{S}-0^{\circ} 5^{\circ} 32^{\prime} 11.1^{\prime \prime} \mathrm{W}$, respectivamente. Em seguida os frutos foram levados para o Laboratório de Análise de Semen- 
tes, da Universidade Federal de Mato Grosso, Campus de Sinop, onde foram extraídas as sementes, realizados o beneficiamento e os testes.

Antes de iniciar os testes de germinação foi determinado o teor de aguadas sementes, de acordo com Brasil (2009), com quatro repetições de dez sementes cada. As sementes de $P$. multijuga apresentavam teor de água de 7,84\%, P. pendula $9,70 \%$ e $P$. platycephala possuíam $11,76 \%$.

Foram testados quatro tratamentos diferentes para superar a dormência causada pela impermeabilidade do tegumento: T1 - escarificação mecânica em esmeril elétrico no lado oposto ao do embrião; T2 - escarificação mecânica em esmeril elétrico no lado oposto ao do embrião seguido por imersão em água corrente por 24h; - T3 - escarificação com ácido sulfúrico $98 \%$ por $10 \mathrm{~min}$.; T4 - imersão em água a $80^{\circ} \mathrm{C}$ por $10 \mathrm{~min}$; e o controle (T5). Posteriormente aos tratamentos, as sementes foram desinfetadas com hipoclorito de sódio a $2 \%$ por 3 min., seguida de lavagem com água destilada, e após imersas em fungicida Maxim a $2 \%$ por $10 \mathrm{~min}$.

As sementes de $P$. pendula e $P$. platycephala foram colocadas para germinar em placas de Petri com quatro repetições de 25 sementes, enquanto que as de $P$. multijuga foram acondicionadas em caixas gerbox, com oito repetições de dez sementes, por apresentarem um tamanho maior. Ambos os recipientes continham ágar a $0,7 \%$ e foram armazenadas em câmaras de germinação tipo B.O.D. (BOD) em três temperaturas diferentes: $25^{\circ} \mathrm{C}$ e $30^{\circ} \mathrm{C}$ constantes, e $25-30^{\circ} \mathrm{C}$ alternadas, todas com fotoperíodo de 12 horas.

As avaliações para controle da germinação foram realizadas diariamente, tendo como critério para avaliação a protrusão da raiz primária $(2 \mathrm{~mm})$. O controle foi avaliado por 90 dias e os demais tratamentos por 40 dias, uma vez que não há um critério estabelecido sobre o tempo que se deve esperar para que a semente germine, antes de considerá-la como dormente (Cardoso, 2009). Baskin \& Baskin (1998) sugerem que o teste de germinação seja avaliado por duas semanas, podendo ser prorrogado para quatro se a porcentagem de germinação continuar aumentando.

O delineamento experimental empregado foi o Delineamento em Blocos Casualizados (DBC) em esquema fatorial (5X3), com cinco tratamentos de superação de dormência e três temperaturas. Os dados foram avaliados através da porcentagem final de germinação e o índice de velocidade de germinação (IVG) proposto por Maguire (1962). Os dados da porcentagem de germinação e IVG foram transformados em $\sqrt{ } \mathrm{x}$, porém nas tabelas são apresentados os dados originais. Os resultados foram submetidos à análise de variância $(f=0,05)$ e as médias comparadas pelo teste Tukey a $5 \%$ de probabilidade.

\section{Resultados e Discussão}

Com exceção da $P$. multijuga, a porcentagem de germinação das demais espécies apresentaram interação significativa para os dois fatores avaliados (temperatura e método de superação de dormência)conforme pode ser observado na Tabela 1, indicando que existe pelo menos uma combinação ideal entre esses fatores que otimiza a porcentagem de germinação (Alves et al, 2002) e o aumento no IVG. Essas interações são importantes para demonstrar que o método de superação de dormência pode refletir em porcentagens de germinação e IVG diferentes quando submetidas a condições de temperaturas distintas. Essa interação também foi encontrada para sementes de Ocotea corymbosa (Bilia et al, 1998) e Adenanthera pavonina (Silva et al, 2009). Para sementes de Caesalpinia leiostachya, Biruel et al (2007) não encontraram interação significativa entre temperatura e o método de superação de dormência testados. Isso demonstra que cada espécie possui suas especificidades, havendo interações ou não com os diferentes métodos de superação de dormência e as temperaturas utilizadas.

Tabela 1 - Porcentagem de germinação de sementes de Parkia platycephala, Parkia pendula e Parkia multijuga submetidas a diferentes métodos de superação de dormência e temperatura.

\begin{tabular}{|c|c|c|c|c|c|c|c|}
\hline \multirow{3}{*}{$\begin{array}{l}\text { Tratamento para } \\
\text { superação de } \\
\text { dormência }\end{array}$} & \multicolumn{7}{|c|}{ Porcentagem de germinação } \\
\hline & \multicolumn{3}{|c|}{ Parkia platycephala } & \multicolumn{3}{|c|}{ Parkia pendula } & \multirow{2}{*}{$\begin{array}{l}\text { Parkia } \\
\text { multijuga }\end{array}$} \\
\hline & $25^{\circ} \mathrm{C}$ & $30^{\circ} \mathrm{C}$ & $25-30^{\circ} \mathrm{C}$ & $25^{\circ} \mathrm{C}$ & $30^{\circ} \mathrm{C}$ & $25-30^{\circ} \mathrm{C}$ & \\
\hline $\begin{array}{l}\text { Escarificação } \\
\text { mecânica }\end{array}$ & $100 \pm 0,0 \mathrm{aA}$ & $100 \pm 0,0 \mathrm{aA}$ & $98 \pm 1,15$ aA & $98 \pm 3,7 a A$ & $100 \pm 0,0 a A$ & $100 \pm 0,0 \mathrm{aA}$ & $88,75 \pm 1,5 a$ \\
\hline $\begin{array}{l}\text { Escarificação me- } \\
\text { cânica }+ \text { imersão } \\
\text { água } 24 \mathrm{~h}\end{array}$ & $82 \pm 2,5 \mathrm{bA}$ & $91 \pm 1,91 \mathrm{aA}$ & $88 \pm 2,82 b A$ & $80 \pm 3,38 \mathrm{bB}$ & $89 \pm 1,0 \mathrm{bAB}$ & $95 \pm 1,0$ aA & $95,83 \pm 1,3 a$ \\
\hline $\mathrm{H}_{2} \mathrm{SO}_{4} 10 \mathrm{~min}$ & $85 \pm 5,7 \mathrm{bB}$ & $94 \pm 2,58 \mathrm{aAB}$ & $97 \pm 1,91$ aA & $15 \pm 1,91 \mathrm{cB}$ & $28 \pm 1,63 \mathrm{bA}$ & $31 \pm 1,0 \mathrm{bA}$ & $94,16 \pm 1,3 a$ \\
\hline $\begin{array}{l}\mathrm{H}_{2} \mathrm{O} 80^{\circ} \mathrm{C} \text { por } \\
10 \mathrm{~min}\end{array}$ & $4 \pm 0,0 \mathrm{dA}$ & $5 \pm 1,0 \mathrm{cA}$ & $5 \pm 1,0 \mathrm{cA}$ & $5 \pm 0,92 \mathrm{~dB}$ & $11 \pm 1,0 \mathrm{cA}$ & $6 \pm 1,15 \mathrm{cB}$ & $16,25 \pm 1,6 c$ \\
\hline Controle & $30 \pm 1,1 \mathrm{cAB}$ & $32 \pm 1,63 \mathrm{bA}$ & $25 \pm 2,51 \mathrm{cB}$ & $6 \pm 1,15 \mathrm{~dB}$ & $11 \pm 1,0 \mathrm{cA}$ & $8 \pm 0,0 \mathrm{cAB}$ & $28,33 \pm 1,6 b$ \\
\hline CV (\%) & & 4,25 & & & 5,19 & & 7,80 \\
\hline
\end{tabular}

As médias seguidas pela mesma letra, minúscula na coluna e maiúscula na linha, não diferem entre si pelo Teste de Tukey ao nível de $5 \%$ de probabilidade 
O tratamento $T 1$, onde foi utilizado escarificação com lixa, foi eficiente para a superação de dormência das três espécies em todas as temperaturas testadas (Tabela 1 ). O sucesso do método aplicado pode ter ocorrido em função da ruptura da barreira física que impede a troca de gases e água entre o embrião e o meio externo, sendo então observado uma taxa maior de germinação e IVG. O T2 apresentou porcentagem de germinação estatisticamente igual ao T1 para $P$. platycephala na temperatura de $30^{\circ} \mathrm{C}$ e para P. pendula a $25-30^{\circ} \mathrm{C}$ (Tabela 1). Os tratamentos T2 e T3 também foram eficientes para a $P$. multijuga (Tabela 1). Porém, quando analisado o IVG, para a $P$. multijuga o T2 foi superior aos demais (Tabela 2). Isso comprova que esses métodos quando aplicados causam ruptura e desarranjo da estrutura do tegumento, facilitando assim o processo de embebição e a ativação do processo de germinação. O IVG apresentado pelo T1 foi significante em todas as temperaturas para $P$. pendula e, com exceção de $25^{\circ} \mathrm{C}$, para $P$. platycephala (Tabela 2). Os tratamentos T1 e T2 foram superiores aos demais, quando analisado a porcentagem de germinação, pelo fato de terem produzido o mesmo efeito dos demais tratamentos, de forma a não comprometer aqualidade fisiológica das sementes (Nascimento et al, 2009) e sem prejudicar a integridade do embrião, superando a dormência e aumentando a velocidade de germinação. O T4 apresentou menor desempenho, com resultados semelhantes e/ou inferiores ao controle para todas as características avaliadas nas três espécies, superação e dormência e IVG (Tabelas 1 e 2).

Tabela 2 - Índice de velocidade de germinação (IVG) de Parkia platycephala, Parkia pendula e Parkia multijuga submetidas a diferentes métodos de superação de dormência e temperatura.

\begin{tabular}{|c|c|c|c|c|c|c|c|c|c|}
\hline \multirow{3}{*}{$\begin{array}{l}\text { Tratamento } \\
\text { para } \\
\text { superação } \\
\text { de } \\
\text { dormência }\end{array}$} & \multicolumn{9}{|c|}{ Índice de Velocidade de Germinação - IVG } \\
\hline & \multicolumn{3}{|c|}{ Parkia platycephala } & \multicolumn{3}{|c|}{ Parkia pendula } & \multicolumn{3}{|c|}{ Parkia multijuga } \\
\hline & $25^{\circ} \mathrm{C}$ & $30^{\circ} \mathrm{C}$ & $25-30^{\circ} \mathrm{C}$ & $25^{\circ} \mathrm{C}$ & $30^{\circ} \mathrm{C}$ & $25-30^{\circ} \mathrm{C}$ & $25^{\circ} \mathrm{C}$ & $30^{\circ} \mathrm{C}$ & $25-30^{\circ} \mathrm{C}$ \\
\hline $\begin{array}{l}\text { Escarificação } \\
\text { mecânica }\end{array}$ & $14,16 \pm 0,2 \mathrm{aB}$ & $19,69 \pm 0,5 \mathrm{aA}$ & $17,25 \pm 0,8 \mathrm{aA}$ & $15,95 \pm 0,6 \mathrm{aA}$ & $18,45 \pm 0,2 \mathrm{aA}$ & $15,37 \pm 0,5 \mathrm{aA}$ & $0,94 \pm 0,02 c B$ & $1,26 \pm 0,03 c A$ & $1,1 \pm 0,09 \mathrm{bAB}$ \\
\hline $\begin{array}{l}\text { Escarificação } \\
\text { mecânica + } \\
\text { imersão em } \\
\text { água } 24 \text { h }\end{array}$ & $14,37 \pm 0,4 a A$ & $15,17 \pm 0,4 \mathrm{bA}$ & $14,92 \pm 0,4 a A$ & $12,98 \pm 0,6 a \mathrm{~B}$ & $13,52 \pm 0,3 \mathrm{bB}$ & $17,03 \pm 0,2 \mathrm{aA}$ & $1,91 \pm 0,1 \mathrm{aB}$ & $2,29 \pm 0,09 \mathrm{aA}$ & $2,43 \pm 0,08 \mathrm{aA}$ \\
\hline $\begin{array}{l}\mathrm{H}_{2} \mathrm{SO}_{4} \\
10 \mathrm{~min}\end{array}$ & $7,33 \pm 0,2 \mathrm{bB}$ & $9,78 \pm 1,0 \mathrm{CA}$ & $8,28 \pm 1,3 \mathrm{bAB}$ & $0,49 \pm 0,1 \mathrm{bcA}$ & $1,81 \pm 0,2 \mathrm{CA}$ & $1,37 \pm 0,6 \mathrm{bAB}$ & $1,50 \pm 0,09 \mathrm{bAB}$ & $1,76 \pm 0,05 \mathrm{bA}$ & $1,23 \pm 0,1 \mathrm{bB}$ \\
\hline $\begin{array}{l}\mathrm{H}_{2} \mathrm{O} 80^{\circ} \mathrm{C} \\
\text { por } 10 \mathrm{~min}\end{array}$ & $0,08 \pm 0,0 \mathrm{dA}$ & $0,09 \pm 0,02 \mathrm{eA}$ & $0,08 \pm 0,02 \mathrm{dA}$ & $0,85 \pm 0,2 \mathrm{bA}$ & $1,11 \pm 0,2 \mathrm{cA}$ & $0,79 \pm 0,2 \mathrm{bcA}$ & $0,05 \pm 0,01 \mathrm{dA}$ & $0,13 \pm 0,04 d A$ & $0,11 \pm 0,02 \mathrm{cA}$ \\
\hline Controle & $1,9 \pm 0,3 \mathrm{cA}$ & $2,19 \pm 0,1 \mathrm{dA}$ & $1,06 \pm 0,1 \mathrm{cB}$ & $0,09 \pm 0,03 \mathrm{cA}$ & $0,12 \pm 0,04 \mathrm{~d} A$ & $0,23 \pm 0,1 \mathrm{CA}$ & $0,12 \pm 0,03 \mathrm{~d} A$ & $0,17 \pm 0,02 \mathrm{dA}$ & $0,20 \pm 0,02 c A$ \\
\hline CV (\%) & & 7,62 & & & 12,63 & & & 12,44 & \\
\hline
\end{tabular}

As médias seguidas pela mesma letra, minúscula na coluna e maiúscula na linha, não diferem entre si pelo Teste de Tukey ao nível de 5\% de probabilidade.

A escarificação mecânica tem se mostrado um eficiente método para superação de dormência de espécies florestais como destacado por Fowler \& Bianchetti (2000, em especial as leguminosas, método este que proporcionou melhores resultados para as espécies estudadas, com a menor porcentagem de germinação dentre as espécies estudadas de $88,75 \%$ para a $P$. multijuga (Tabela 1).

Para as demais espécies não houve diferença estatística entre as temperaturas quando adotado este método de superação. Este é um procedimento fácil e rápido para a superação de dormência das sementes, o qual possibilita o processo de embebição, resultando em uma germinação mais rápida e uniforme. Oliveira et al (2012), avaliando tratamentos pré-germinativos em sementes de Samanea tubulosa Bentham encontraram meIhores resultados com o desponte das sementes (escarificação). Espécies de Adesmia DC, estudadas por Tedesco et al (2001) também apresentaram melhores resultados de germinação através da escarificação mecânica.

A escarificação mecânica e química provocam fissuras no tegumento, aumentando a permeabilidade e permitindo a embebição (Alves et al, 2007) e consequentemente, favorecendo o início da germinação, por isso o T1, T2 e T3 apresentaram-se eficientes para a superação da dormência de $P$. multijuga, não havendo diferenças estatís- 
ticas entre eles (Tabela 1). Porém, quando analisado o IVG (Tabela 2), o T2 se mostrou superior aos demais, proporcionando uma germinação mais rápida e uniforme em todas as temperaturas testadas, obtendo um resultado mais satisfatório nas temperaturas de $30^{\circ} \mathrm{C}$ e $25-30^{\circ} \mathrm{C}$, sendo essas possíveis de serem consideradas como faixa de temperaturas ótimas para a espécie, comprovado pelo IVG, onde houve maior taxa de germinação em menor espaço de tempo. A grande quantidade de sementes de $P$. pendula que permaneceram intactas provenientes do tratamento T3 (Tabela 1) evidencia a baixa eficiência do tratamento, necessitando, portanto, de mais estudos relacionados ao tempo de escarificação com o ácido sulfúrico. Upreti \& Dhar (1997) citam dois motivos para que o ácido sulfúrico não tenha causado efeito: o ácido não desgastou suficientemente o revestimento da semente para superar a dormência ou penetrou na parte interna danificando ou matando o embrião. No caso das sementes de $P$. pendula é provável que o tempo de imersão não tenha sido suficiente para causar dano no tegumento e proporcionar a embebição e posterior germinação, uma vez que a maioria das sementes permaneceu sem embebição aparente. Porém, a escarificação com o ácido sulfúrico por dez minutos favoreceu a germinação das sementes de $P$. multijuga e $P$. platycephala, sendo que para esta, quando em temperatura de $30^{\circ} \mathrm{C}$ e $25-30^{\circ} \mathrm{C}$ não apresentou diferença estatística se comparada ao T1 (Tabelas 1). No entanto, quando analisado o IVG, o T3 mostrou-se inferior ao $\mathrm{T} 1$ e T2. Isso porque mesmo rompendo a barreira física provocada pelo tegumento, a absorção de água foi mais lenta em relação aos tratamentos T1 e T2, fazendo com que as sementes germinassem de forma mais lenta e irregular (Tabela 2). A eficiência do ácido sulfúrico na superação da dormência em sementes está relacionada com a espécie e o tempo de exposição da semente ao ácido (Cruz et al, 2001). Eira et al (1993) concluíram que o tratamento com ácido sulfúrico nos tempos de 15,30, 60 e 90 minutos superou a dormência de Enterolobium contortisiliquum. Resultados semelhantes foram encontrados para Piptadenia moniliformis (Azeredo et al, 2010), Mimosa foliolosa (Silveira \& Fernandes, 2006) e Ormosia nitida (Lopes et al, 2006).

Ainda que a porcentagem de germinação do controle (T5) das três espécies tenha sido baixa, os resultados foram superiores quando comparados com o tratamento T4, o que demonstra que o mesmo não foi eficiente para a superação de dormência das três espécies em todas as temperaturas testadas (Tabela 1). As sementes de $P$. multijuga e $P$. pendula permaneceram com a absorção de água muito baixa, mantendo-se intactas, sem alterações morfológicas aparente. É provável que o tempo de imersão na água quente tenha sido insuficiente para romper o tegumento e superar a dormência das sementes. As sementes de $P$. platycephala apresentaram vestígios de deterioração, uma vez que foi observado extravasamento de exsudatos e presença de bactérias, podendo ter ocorrido dano ao embrião em função do tempo de exposição em excesso à água quente $\left(80^{\circ} \mathrm{C}\right)$. Esses resultados demonstram que a dormência é variável entre as espécies, conforme a espessura do tegumento e também devido à presença de substâncias impermeáveis (Oliveira et al, 2012). Resultados negativos para uso da água quente também foi encontrado por Bruno et al (2001) para as sementes de Mimosa caesalpiniaefolia. Porém, Oliveira, Davide \& CarvaIho (2003) concluíram que a imersão em água quente favorece a germinação de Peltophorum dubium. Portanto, a aplicabilidade e eficiência desses tratamentos dependem do tipo e da intensidade da dormência que varia entre as espécies (Bruno et al, 2001).

A temperatura também é um fator determinante para o processo germinativo, podendo afetar o total, a velocidade e uniformidade da germinação (Carvalho \& Nakagawa, 2000). Brancalion et al (2010) citam que a temperatura de $25^{\circ} \mathrm{C}$ é ótima para a germinação das sementes da maioria das espécies florestais brasileiras, seguida por $30^{\circ} \mathrm{C}$, em especial na Amazônia, bioma de coleta das sementes utilizadas. Nessa faixa de temperatura ótima, ocorre a maior porcentagem de germinação no menor espaço de tempo (Albrecht, Albuquerque \& Silva, 1986). As sementes de $P$. multijuga, analisando a porcentagem de germinação e o IVG, apresentaram melhor desempenho na temperatura de $30^{\circ} \mathrm{C}$ (Tabela 2 e 3). O mesmo foi encontrado para as sementes de $P$. pendula e P. platycephala (Tabelas 1 e 2). Estudos apontam que a temperatura interage com os hormônios vegetais, de forma a alterar seus níveis endógenos (Bewley \& Black, 1982) afetando as reações bioquímicas que determinam todo o processo germinativo (Floriano, 2004. Por isso é muito importante que seja realizado estudos com diferentes temperaturas associadas aos tratamentos de superação de dormência. De acordo com Carvalho \& Nakagawa (2000), temperaturas inferiores ou superiores à ótima tendem a reduzir a velocidade do processo germinativo, expondo as plântulas por maior período a fatores adversos, o que pode levar à redução no total de germinação.

Tabela 3 - Porcentagem de germinação de sementes de Parkia multijuga submetidas a diferentes temperaturas.

\begin{tabular}{cc}
\hline Temperatura & Germinação (\%) \\
\hline $\mathbf{2 5}^{\circ} \mathbf{C}$ & $62,50 \pm \mathrm{b}$ \\
$\mathbf{3 0}^{\circ} \mathbf{C}$ & $66,25 \pm \mathrm{a}$ \\
$\mathbf{2 5 / 3 0 ^ { \circ } \mathbf { C }}$ & $65,25 \pm \mathrm{ab}$ \\
\hline $\mathbf{C V}(\%)$ & 7,80 \\
\hline
\end{tabular}

As médias seguidas pela mesma letra não diferem estatisticamente entre si pelo Teste de Tukey ao nível de 5\% de probabilidade. 


\section{Conclusão}

Com base nos resultados obtidos recomenda-se utilizar a escarificação mecânica em esmeril elétrico no lado oposto ao do embrião como método de superação de dormência das sementes de $P$. pendula e $P$. platycephala. Para sementes de $P$. multijuga recomenda-se a escarificação mecânica em esmeril elétrico no lado oposto ao do embrião seguido por imersão em água corrente por $24 \mathrm{~h}$. A temperatura de $30^{\circ} \mathrm{C}$ é a mais indicada para as três espécies.

\section{Agradecimentos}

À Fapemat pelo apoio financeiro para desenvolvimento da pesquisa. Aos Professores Dr. Evaldo Martins Pires (UFMT) e Dr. Douglas dos Santos Pina (UFMT) pelo auxílio nas análises estatísticas.

\section{ReferênCIAS}

Albrecht, J.M.F.; M.C.L.F. Albuquerque \& V.S.M. Silva. 1986. Influência da temperatura e do tipo de substrato na germinação de sementes de cerejeira. Revista Brasileira de Sementes 8(1):49-55.

Alves, E.U.; E.A. Cardoso; R.L.A. Bruno; A.U. Alves; E.A. Galindo \& J.M.B. Junior. 2007. Superação da dormência em sementes de Caesalpinia pyramidalis Tul. Revista Árvore 31(3):405-415.

Alves, E.U.; R.C.Paula; A.P. Oliveira; R.L.A. Bruno \& A.A. Diniz. 2002. Germinação de sementes de Mimosa caesalpiniaefolia Benth. em diferentes substratos e temperaturas. Revista Brasileira de Sementes. 24(1):169178.

Alves, M.C.S.; S.M. Filho; M.A.Neto \& E.M.Teófilo. 2000. SUPERAÇÃO DA DORMÊNCIA EM SEMENTES DE Bauhinia monandra e $B$. ungulata. Revista Brasileira de Sementes 22(2):139-144.

Azeredo, G.A. 2010. Superação de dormência de sementes de Piptadenia moniliformis Benth. Revista Brasileira de Sementes 32(2):049-058.

Azeredo, G.A.; R.C. Paula; S.V. Valeri \& F.V. Moro. 2010. Superaçao de dormência de sementes de Peptadenia moniliformis Benth. Revista Brasileira de Sementes 32(2):49-58.

Bacha, C.J.C. 2008. Análise da Evolução do Reflorestamento no Brasil. Revista de Economia Agrícola 55(2):5-24.
Baskin, C.C. \& J.M. Baskin. 1998. Seeds: ecology, biogeography and evolution of dormancy and germination. Academic Press, San Diego. 666p.

Bewley, J.D \& M. Black. 1982. Physiology and biochemistry of seeds in relation to germination - Viability, dormancy and environmental control, v. 2. Berlim: Springer-Verlag. 375 p.

Bilia, D.A.C.; C.J. Barbedo \& A.M. Maluf. 1998. Germinação de diásporos de canela ( (Ocotea corymbosa (Meissn.) Mez - Lauraceae) em função da temperatura, do substrato e da dormência. Revista Brasileira de Sementes 20(1):189-194.

Biruel, R.P.; I.B. Aguiar \& R.C. Paula. 2007. Germinação de sementes de pau ferro submetidas a diferentes condições de armazenamento, escarificação química, temperatura e luz. Revista Brasileira de Sementes 29(3):151-159.

Brancalion, P.H.S.; A.D.L.C. Novembre \& R.R. Rodrigues. 2010. Temperatura ótima para germinação de sementes de espécies arbóreas brasileiras. Revista Brasileira de Sementes 32(4):15-21.

Bruno, R.L.A.; E.U. Alves; A.P. Oliveira \& R.C. Paula. 2001. Tratamento pré-germinativos para superar a dormência de sementes de Mimosa caesalpiniaefolia Benth. Revista Brasileira de Sementes 23(2):136-143.

Cardoso, V.J.M. 2004. Dormência: estabelecimento do processo. p.95-108 In: Borghetti, F. \& A.G. Ferreira (org). Germinação: do básico ao aplicado. Porto Alegre: Artmed.

Cardoso, V.J.M. 2009. Conceito e classificação de dormência. Oecologia Brasiliensis 13:619-631.

Carvalho, N. M; Nakagawa. J. Sementes: ciência, tecnologia e produção. $4^{\circ} \mathrm{Ed}$. Jaboticabal: Funep, 2000. 588p.

Cruz, E.D.; J.E.U. Carvalho \& N.V.M. Leão. 2001. Métodos para superação da dormência e biometria de frutos e sementes de $\mathrm{Pa}$ rkia nitida Miguel. (Leguminosae - Mimosoideae). Acta Amazonica 31(2):167-177.

Eira, M.T.S.; R.W.A. Freitas \& C.M.C. Mello. 1993. Superação da dormência de sementes de Enterolobium contortisiliquum (Vell.) Morong. - Leguminosae. Revista Brasileira de Sementes 15(2):177-181.

Escobar, T.A.; V.M. Pedroso; R.N. Bonow \& E.B.Schwengber. 2010. Superaão de dor- 
mência e temperaturas para germinação de sementes de Acacia caven (Mol.) Mol. (espinilho). Revista Brasileira de Sementes 32(2):124-130.

Finch-Savage, W.E \& G. Leubner-Metzger. 2006. Seed dormancy and the control of germination. New Phytologist 171:501-523.

Floriano, E.P. 2004. Germinação e dormência de sementes florestais. v.2. Santa Rosa. 19.

Fowler, J.A.P. \& A. Biancheti. 2000. Dormência em sementes florestais. Colombo: Embrapa Florestas. 27p.

Galeão R. R.; J.O.P. Carvalho; J.A.G. Yared; L.C.T. Marques \& P.P.C. Filho. 2006. Diagnóstico dos projetos de reposição florestal do estado do Pará. Revista Ciências Agrárias 45:101-120.

Hermansen, L. A.; M.L. Duryea; S.H. West; T.L. White \& M.M. Malavasi. 2000. Records Pretreatments to overcome seed coat dormancy in Dimorphandra mollis. Seed Science and Technology 28(3):581-595.

Hopkins, H.C. 1986. Parkia (Leguminosae: Mimosoideae). In Flora Neotrópica 43. New York Botanical Garden, 124p.

Lopes, J.C.; P.C. Dias \& C.M.P. Azevedo. 2006. Tratamentos para acelerar a germinação e reduzir a deterioração das sementes de Ormosia nitida Vog. Revista Árvore 30(2):171-177.

Maguire, J. D. 1986 . Speed of germination aid in selection and evaluation for seeding emergence and vigor. Crop Science 2(2)176-177.

Melo, M.G.G.; M.S. Mendonça; P. Nazário \& A.M.S. Mendes. 2011. Superação de dormência em sementes de três espécies de Parkia spp. Revista Brasileira de Semente 33(3):533-542.

Ministério da Agricultura e da Reforma Agrária. Regras para análise de sementes. Brasília : Mapa/ACS, 2009. 399p.

Ministério da Agricultura e Pesquisa Agropecuária. (www.agricultura.gov.br) último acesso em 27 de abril de 2013.

Miranda, M.C.P.; A.R. Castelo; D.L.C. Miranda \& E.V. Rondon. 2012. Propriedade físicas e mecênicas da madeira de Parkia gigantocarpa DUCKE. Ciência da Madeira (Braz. J. Wood Sci.) 03(2):55-65.

Nascimento, I.L.; E.U. Alves; R.L.A. Bruno; E.P. Gonçalves; P.N.Q. Colares
\& M.S. Medeiros. 2009. Revista Árvore 33(1):35-45.

Netto, D. A. M. 1994. Germinação de sementes de pau-de-balsa (Ochroma pyramidale (Cav.) Urb.) - Bombacaceae. Revista Brasileira de Sementes, 16(2):159-162.

Oliveira, L.M.; A.C. Davide \& M.L.M. Carvalho. 2003. Avaliação de métodos para quebra da dormência e para a desinfestação de sementes de canafístula (Peltophorum dibuim (Sprengel)) Taubert. Revista Árvore 27(5):597-603.

Oliveira, L.M.; R.L.A. Bruno; E.U. Alves; D.M.Sousa \& A.P. Andrade. 2012. Tratamentos pré-germinativos em sementes de Samanea tubulosa Benthan - (Leguminoseae - Mimosoideae). Revista Árvore 36(3):433-440.

Oliveira, M.C.P.; I.D.K. Ferraz \& G.J. Oliveira. 2006. Dispersão e superação da dormência de sementes de Parkia pendula (Willd.) Walp. (visgueiro) na Amazônia Central, Am, Brasil. Hoehnea 33(4):485-493.

Pereira, M.L.; A. Zanon \& M.C. Scheffer.1995. Germinação de sementes de guaco - Mikania glomerata Spreng. (Asteraceae). Horticultura Brasileira 13(1):104.

Pereira, S.A. \& S.A.N. Ferreira. 2010. Superação da dormência em sementes de visgeuiro-do-igapó (Parkia discolor). Acta Amazonica 40(1):151-156.

Pinedo, G.J.P. \& I.D.K.Ferraz. 2008. Hidrocondiocionamento de Parkia pendula [Benth ex Walp]: sementes com dormência física de árvore da Amazônia. Revista Árvore 32(1):39-49.

Ribas, L.L.F; L.C. Fossati \& A.C. Noguria. 1996. Superação de dormência de sementes de Mimosa bimucronata (DC.) O. Kuntze (Maricá). Revista Brasileira de Sementes, 18(1):98-101.

Rosseto, J.; M.C.F. Albuquerque; R.M.R. Neto \& I.C.O. Silva. 2009. Germinação de sementes de Parkia pendula (wiIld.) Benth. Ex Walp. (Fabaceae) em diferentes temperaturas. Revista Árvore 33(1):47-55.

Schmidt, L. 2000. Guide to handling of tropical and subtropical forest seed. Denmark: Danida Forest Seed Centree, 511p.

Silva, A.I.S.; V.B.Corte; M.D. Pereira; G.R.F.Cuzzuol \& I.T.A. Leite. 2009. Efeito 
da temperatura e de tratamentos pré-germinativos na germinação de sementes de Adenanthera pavonina L. Semina: Ciências Agrárias 30(4):815-824.

Silveira, F.A.O. \& G.W. Fernandes. 2006. Effect of light, temperature and scarification on the germination of Mimosa foliolosa (Leguminosae) seeds. Seed Science \& Technology, 34:585-592.

Tedesco, S.B.; M.O. Stefanello; M.T. SchifinoWittmann; A. Battistin \& M. Dall'Agnol. 2001. Superação de dormência em sementes de espécies de Adesmia DC. (Leguminosae). Revista Brasileira de Agrociências 7(2):89-92.
Upreti, J. \& U. Dhar. 1997. Study on seed germination of a leguminous liana - Bauhinia vahlii Wight and Arnott. Seed Science and Technology.

Varela,V.P.; P.A.N. Aquino \& C.P. Azevedo. 1987. Tratamentos pré-germinativos em sementes de espécies florestais da Amazônia. III. Faveira-arara-tucupi (Parkia decussata Ducke) - Leguminoseae. Acta Amazônica 16 (17:557-562.

Recebido em 15.VII.2013

Aceito em 02.X.2013 